Research Article

\title{
Calculation of Friction Force for Slurry Pipe Jacking considering Soil-Slurry-Pipe Interaction
}

\author{
Yichao Ye (D), Limin Peng, Weichao Yang (D), Yang Zou, and Chengyong Cao \\ School of Civil Engineering, Central South University, Changsha 410075, China \\ Correspondence should be addressed to Weichao Yang; weic_yang@163.com
}

Received 4 October 2019; Revised 23 March 2020; Accepted 12 May 2020; Published 9 June 2020

Academic Editor: Claudia Vitone

Copyright (c) 2020 Yichao Ye et al. This is an open access article distributed under the Creative Commons Attribution License, which permits unrestricted use, distribution, and reproduction in any medium, provided the original work is properly cited.

\begin{abstract}
This paper aims to provide a new approach to predict the friction resistance of slurry pipe jacking. Friction force usually constitutes the main component of jacking force. It can be calculated by multiplying an effective friction coefficient and the normal force acting on the external surface of the pipe. This effective friction coefficient is introduced to reflect the effect of contact state of pipe soil slurry, highly affected by the effect of lubrication and the interaction of pipe soil slurry. Firstly, by making some reasonable assumptions, the analytical formula of the effective friction coefficient is obtained, in which the critical quantity of contact (contact angle or width) is calculated by using the Persson contact model. Then, the analytical formula of normal force of circular pipeline is derived, which needs to determine the vertical soil pressure. To allow for a better prediction, three typical silo models are introduced and compared. Finally, a method for calculating the friction resistance of slurry pipe jacking is established. The main difference from the existing method is that this method takes into full consideration the influence of lubrication, soil properties (such as internal friction angle, cohesion, and void ratio), and design parameters (such as buried depth, overcut, and pipe diameter). By using reasonable silo models, the predicted results are in good agreement with the measured values collected from 10 in situ cases, which proves that the new approach can provide accuracy prediction of friction resistance for slurry pipe jacking with various soil conditions, and it may help for better future design and less construction costs.
\end{abstract}

\section{Introduction}

Pipe jacking is defined as a trenchless excavation technique which employs hydraulic jacks to push specially made pipes through the ground behind a jacking machine, from a drive shaft to a reception shaft. Due to its short time limit, high security, low environmental effect, and little traffic disturbance, it has been widely used in the construction of infrastructures of the traffic and transportation system in the city $[1,2]$. In pipe jacking, the jacking force is a critical factor that determines the thickness of pipe and reaction wall, type of jacking machine, and lubricant requirements, which is directly related to the structural safety and construction cost. Friction resistance is the main component of jacking force. Application of lubricant such as bentonite slurry in pipe jacking (that is, the so-called "slurry pipe jacking") is essential to reduce the friction resistance and therefore the jacking force $[3,4]$. However, it does make it more complex for the calculation or prediction of friction resistance due to the change in contact conditions between the pipe and soil.

At present, the main calculation methods of pipe jacking friction resistance can be divided into the following three categories: First, evaluating from construction experiences; for example, China standard GB50268 suggests the average friction resistance to be $3-5 \mathrm{kPa}$ for slurry pipe jacking. Second, to calculate by multiplying a friction coefficient by the earth pressure $[5,6]$. This method for the calculation of Earth pressure assumes that the excavated bore is unstable, and the surrounding soil is in full contact with the whole area of the jacking pipes. Third, the same form with the second one but using the weight of the pipe or the value of the weight of pipe divided by the contact width instead of the Earth pressure. And the Hertzian contact model is usually used to calculate the contact width $[7,8]$. This kind of method in turn assumes that the excavated bore is stable, and the pipeline simply slides along the bottom of the bore due to 
its own weight. For the later two methods, the friction coefficient is also evaluated by experience; for example, Stein suggested it to be $0.1-0.3$ [9]. Some other authors argued that it can be determined by the tangent of the interfriction angle of soil $\varphi$ or $\varphi / 2$ or $\varphi / 3$ or an angle between them $[5,7,10,11]$.

However, the field monitoring results of 12 related projects (see in Table 1) show that the measured friction resistance is $0.5-5 \mathrm{kPa}$ (conversion of $F_{f} /\left(\pi D_{p}\right)$ is required), most of which are less than $3 \mathrm{kPa}$ or even more than half of which are less than $2 \mathrm{kPa}$. There seems that the suggested value in Chinese standard GB50268 (3-5 kPa) is conservative. Relevant authors used one or more of the above calculation models to predict the friction resistance, and most of the results showed that the measured friction resistances were less than the predicted values of the second type model $[5,6]$, but larger than that of the third type model $[5,7,11]$. The reason may be attributed to the total contact hypothesis of the second type model which overestimates the contact area between the pipe and soil, while the third type model is just the opposite for the limiting contact angle of the Hertzian model which is only $30^{\circ}[12]$. In addition, the value of friction coefficient of pipe soil not only fluctuates greatly and experience dominates, but also depends entirely on soil property (internal friction angle) and ignoring the importance of slurry (as discussed later).

In fact, the factors affecting the friction resistance of slurry pipe jacking are far more complex. Chapman and Ichioka [13] counted 47 in situ cases and found that the frictional resistance along the pipe run $\left(F_{f} f\left(\pi D_{p}\right)\right)(\mathrm{kPa})$ is positively correlated with pipe diameter. In addition, PelletBeaucour and Kastner [5] analyzed 6 cases and found that friction resistance is also related to the overcut (or radial clearance), stoppages, and the size of soil particles (or void ratio). According to Terzaghi's silo model (5) [5], the soil pressure has no correlation with the pipe diameter, overcut, or soil void ratio. If the calculation result of soil pressure is reliable, it can be argued that the effective friction coefficient is also affected by these factors.

Therefore, based on the basic principle of tribology and together with the existing silo models and Persson contact model, a new method suitable for calculating the friction resistance of slurry pipe jacking is established in this paper. It can not only reflect the lubrication effect of slurry, but also comprehensively reflect the influence of redial clearance (or overcut), pipe diameter, soil void ratio, etc., on friction resistance.

\section{Calculation Method of Friction Force for Slurry Pipe Jacking}

In tribology, friction force can be uniformly expressed by multiplying a friction coefficient by the vertical force acting on the surface of an object $[5,7]$ :

$$
F_{f}=\mu \cdot N \text {. }
$$

For slurry pipe jacking, $\mu$ is the effective friction coefficient due to the interaction of soil, lubricant, and the pipe and $N$ is the total normal force acting on the pipe of unit length, under the assumption of plane strain, $\mathrm{kN} / \mathrm{m}$. It is not easy to calculate $\mu$ and $N$. Authors and engineers often suffer from two basic problems that which one of the silo models to use and how to determine the value of $\mu$. Particularly, for the second one, as far as the author can know, there seem no good enough calculation formulas or methods to use for slurry pipe jacking.

2.1. Calculation of $N$. If $\sigma_{n}$ represents the normal stress acting on any point of pipes (see in Figure 1), together with the symmetry of the geometry of the problem, the total normal force $N$ acting on the external surface of the pipe per unit length can be uniformly expressed as an integral form:

$$
N=2 \int_{-\pi / 2}^{\pi / 2} \sigma_{n} \frac{D_{p}}{2} \mathrm{~d} \theta
$$

where $D_{p}$ is the external diameter of pipes and $\theta$ is defined as the angle between the corresponding radius line and the horizontal line at each point of the pipe, positive for counterclockwise and negative for clockwise (see in Figure 1).

In general, the surrounding earth pressure can be described by the vertical earth pressure $\sigma_{v}$ and lateral earth pressure $\sigma_{a}$. It is therefore that the normal stress $\sigma_{n}$ can be expressed as (see in Figure 1)

$$
\sigma_{n}=\sigma_{v} \sin \theta+\sigma_{a} \cos \theta .
$$

Substitute (3) in (2), giving that

$$
N=2 \sigma_{v} D_{p}
$$

As known from (4), the normal force $N$ should only be related to the magnitude of vertical soil stress $\sigma_{v}$ acting on the pipe crown. It has to be noted that at the present time by far the most commonly used model for soil pressure calculation is Terzaghi's silo model [5,7]. Terzaghi's theory assumes that the ground above the excavated tunnel is settling along two vertical planes. These displacements are significant enough to produce sliding planes; see Figure 2. The formula of the vertical soil stress on the pipe crown is given by [5]

$$
\sigma_{v}=\frac{b \gamma-2 c}{2 K \tan (\delta)}\left(1-e^{(-2 K \tan (\delta) \cdot h) / b}\right),
$$

where $h$ is the height of cover at pipe crown, $\gamma$ is the unit weight of soil, $c$ is the soil cohesion, $\varphi$ is the internal friction angle of soil, $\delta$ is the friction angle between the pipe and soil, $K$ is the coefficient of soil pressure above the pipe, and $b$ is the influencing width of soil above the pipe ideal silo width.

It is noted that when the height of cover above the pipe is small $(h<b)$, the "vault" effect of the ground, considered by Terzaghi, is neglected and the whole Earth weight is taken into account. By introducing a coefficient $k$ which represents the "vault" effect of the ground, the vertical stress at the pipe crown $\sigma_{v}$ can also be rewritten as 


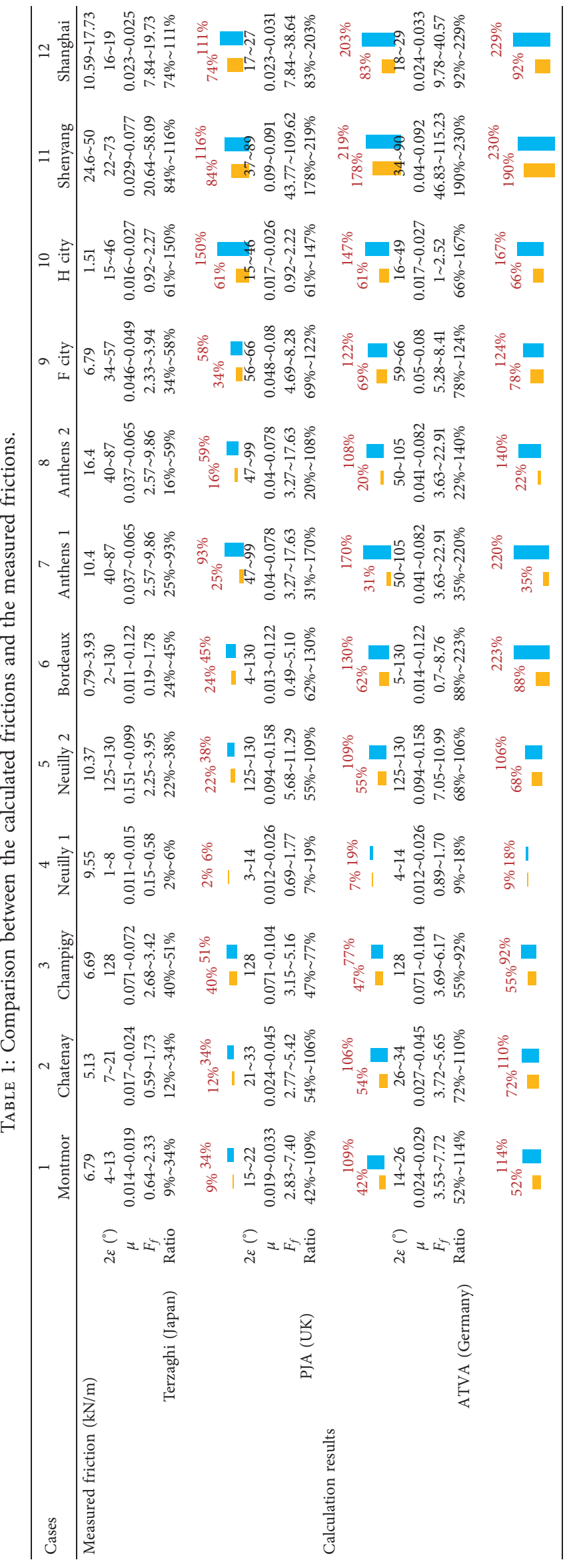




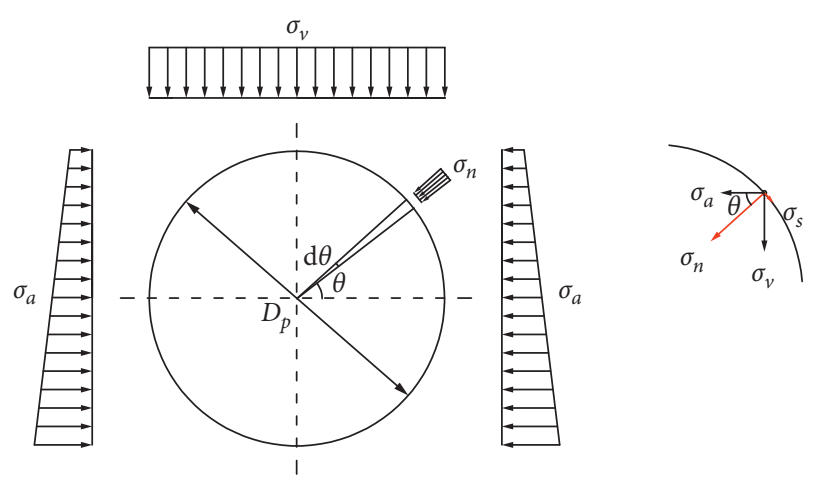

FIGURE 1: Earth pressure and the normal stress acting on the pipe.

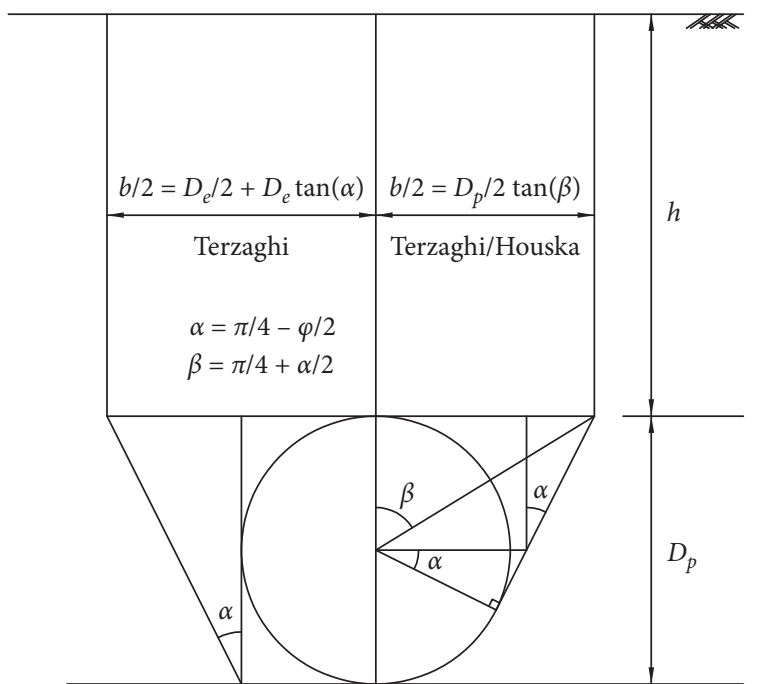

Figure 2: Boundary planes of wedge failure. Terzaghi and Terzaghi/ Houska silo model.

$$
\sigma_{v}=k \gamma h, \begin{cases}k=1, & h<b, \\ k=\frac{1-e^{(-2 K \tan (\delta) \cdot h) / b}}{2 K \tan (\delta)}\left(\frac{b}{h}-\frac{2 c}{\gamma h}\right), & h>b .\end{cases}
$$

The $k$ coefficient is a constant below one. The larger $k$ is, the larger the "vault" effect of the ground will be.

Even though the Terzaghi silo model is widely accepted by the authors from all over the world, but for the determination of empirical parameters (such as $b 、 \delta$, and $K$ ), authors are divided. The most representative modified model is that proposed by UK PJA (Pipe Jacking Association), which assumes another boundary planes of these wedge failures based on Terzaghi/Houska formula; see in Figure 2. And this modified model is also accepted by Germany Standard ATVA 161 but assumes a constant value for the angle of internal friction of $30^{\circ}$. It is obvious that the modified model has a smaller $b$, leading to a larger value of $k$ (or in other wards it weakens the "vault" effect of soil). So the Earth pressure calculated by the modified model is generally larger than that calculated by Terzaghi's initial silo model. The rules to calculate the three parameters, for the three models, were summarized by Pellet-Beaucour and Kastner [5], as given in Table 2.

Specifically, comparison of vertical stresses calculated according to the three different models had been done by Pellet-Beaucour and Kastner. He figured out that the vertical stresses calculated by ATVA 161 model is the largest, while that calculated by Terzaghi initial model is the smallest. However, it is still not convincing to pick out a model to use without checking out with the field data. This work will be carried out in Section 3.

2.2. Calculation of $\mu$. At the present time by far, the friction coefficient is usually considered to be a constant, which can be expressed as $[5,6,10,12,14]$

$$
\mu=\tan (\delta)
$$

It is generally accepted that $\delta=\varphi$ for the calculation of static friction and $\delta=\varphi / 2$ for the calculation of kinematic friction $[6,14]$. But for slurry pipe jacking, the determination of $\delta$ varies from person to person; for example, Barla et al. [12] suggest $\delta$ to be between $\varphi / 2$ and $\varphi$, and Pellet-Beaucour and Kastner, Stein et al. $[5,10]$ suggest $\delta$ to be between $\varphi / 3$ and $\varphi / 2$, depending on the roughness of the pipe-soil interface and the amplitude of motion. As we have discussed above, the range of values seems too large to determine and more importantly the effect of lubrication is absolutely neglected.

In slurry pipe jacking, the use of slurry changes the contact conditions between soil and the pipe. In design philosophy, the overcut should be completely filled with slurry to reduce the friction resistance for maximum efficiency, with no interpenetration or interpenetration terminates in a short time, creating a "filter cake" layer around the cavity and then pressurized to the support pressure required for the soil (see in Figure 3(a)) [15]. In this case, the friction force is only related to the friction coefficient between slurry and the pipe. However, the more general case is that the excavated bore is stable and part of the pipe inevitably comes into contact with soil (see in Figure 3(b)) [3]. The reasons for the occurrence of pipe-soil contact are complex, such as the design and control of grouting amount of slurry, jacking speed, pipeline deviating from the intended line and level, irregular deformation of the surrounding soil, and interpenetration between the soil and slurry. In such a case, the accurate calculation of friction force should take into account contact position, value of contact angle (or width), and contact force. However, for various reasons listed above, it seems impossible and unnecessary to calculate these quantities in a target section of the pipeline. If we focus on the final contact state of pipe soil slurry by ignoring the various factors that lead to it and taking the whole pipeline into consideration, this problem can be greatly simplified by introducing some basic hypotheses:

(i) Contact can occur at any position of a section of the pipeline with the same probability 
TABLE 2: Summary table of assumed model parameters.

\begin{tabular}{|c|c|c|c|}
\hline & Terzaghi (Japan) & ATVA 161 (Germany) & PJA (UK) \\
\hline$B$ & $D_{p}(1+2 \tan ((\pi / 4)-(\phi / 2)))$ & $\sqrt{3} D_{p}$ & $D_{p} \tan ((3 \pi / 8)-(\phi / 4))$ \\
\hline$\Delta$ & $\varphi$ & $\varphi / 2^{P}$ & $\varphi$ \\
\hline K & 1 & 0.5 & $(1-\sin \phi) /(1+\sin \phi)$ \\
\hline
\end{tabular}

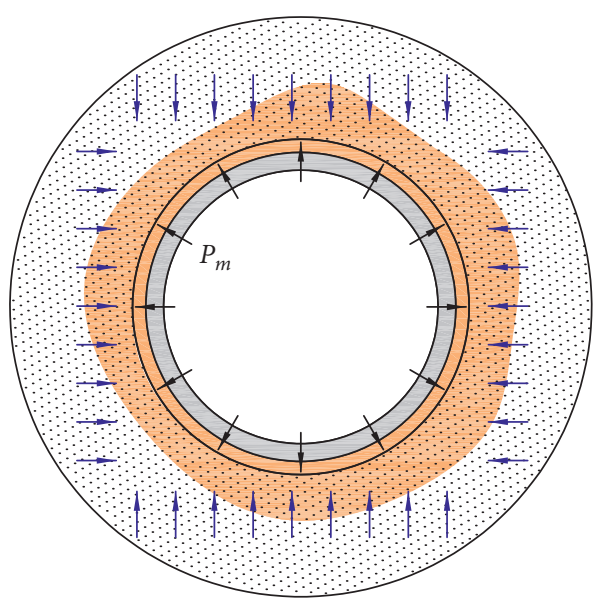

(a)

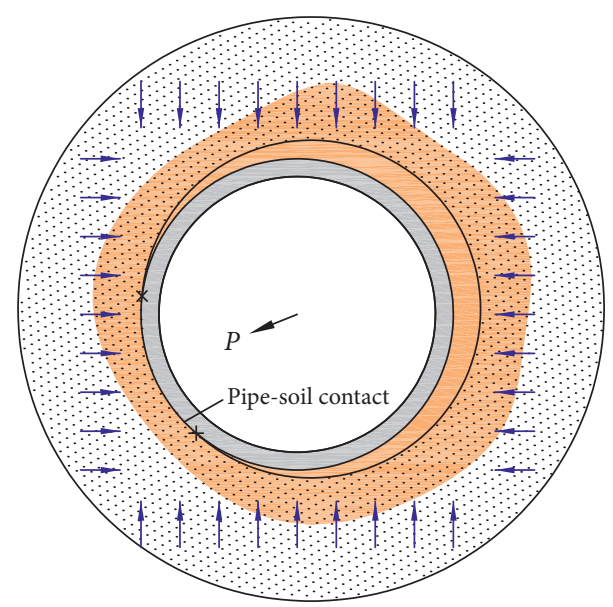

(b)

Figure 3: The contact state of pipe soil slurry: (a) the ideal state; (b) the general state.

(ii) The occurrence of contact does not significantly change the soil pressure in the contact area

(iii) The interpenetration between slurry and soil is quasistatic, and that does not change the geometric structure of soil

(iv) After the slurry injected and filled up the overcut, the slurry pressure can be redistributed and balanced with the soil pressure

Hypotheses (i) and (ii) exactly eliminate the influence of contact position and contact force on the effective friction coefficient. Hypothesis (iii) is for careful consideration; in fact, it will not have a significant impact on the final calculation result.

Generally, the friction force of slurry pipe jacking $F_{f}$ can be divided into the pipe-soil friction force $f_{s}$ and the pipeslurry friction force $f_{m}$ :

$$
\begin{aligned}
& F_{f}=\mu N=f_{s}+f_{m}, \\
& f_{s}=\mu_{s} N_{s}, \\
& f_{m}=\mu_{m} N_{m},
\end{aligned}
$$

where $\mu_{s}(=\tan (\varphi / 2))$ is the coefficient of kinematic friction between soil and the pipe [6], $\mu_{m}$ is the coefficient of kinematic friction between slurry and the pipe, its value can be taken as 0.01 [16], and $N_{s}$ and $N_{m}$ are the total normal force acting on the pipe in the pipe-soil and pipe-slurry contact area, respectively.
According to hypothesis (i), we have the following equations:

$$
\begin{aligned}
N_{s} & =\frac{B_{s}}{C} N=\frac{\varepsilon}{\pi} N, \\
N_{m} & =\frac{B_{m}}{C} N,
\end{aligned}
$$

where $C$ is the external circumference of pipe, $B_{s}$ and $B_{m}$ are the width of contact area between soil and the pipe and that between lubricant slurry and the pipe, respectively, and $\varepsilon$ is the semiangle of contact (as see in Figure 4). It is noted that the value of $\varepsilon$ is roughly supposed to be $\pi / 3$ for any formation [6]; however, there is no evidence to support this conclusion.

By substituting (9)-(12) into (8), after some algebra, the expression of the effective friction coefficient $\mu$ can be written as

$$
\begin{aligned}
\mu & =\mu_{s} \lambda_{s}+\mu_{m} \lambda_{m}, \\
\lambda_{s} & =\frac{B_{s}}{C}=\frac{\varepsilon}{\pi}, \\
\lambda_{m} & =\frac{B_{m}}{C} .
\end{aligned}
$$

According to hypothesis (iii), the relation between $B_{m}$ and $B_{s}$ can be expressed as

$$
B_{m}=C-\frac{B_{s}}{1+e}
$$

where $e$ is the void ratio of soil. 


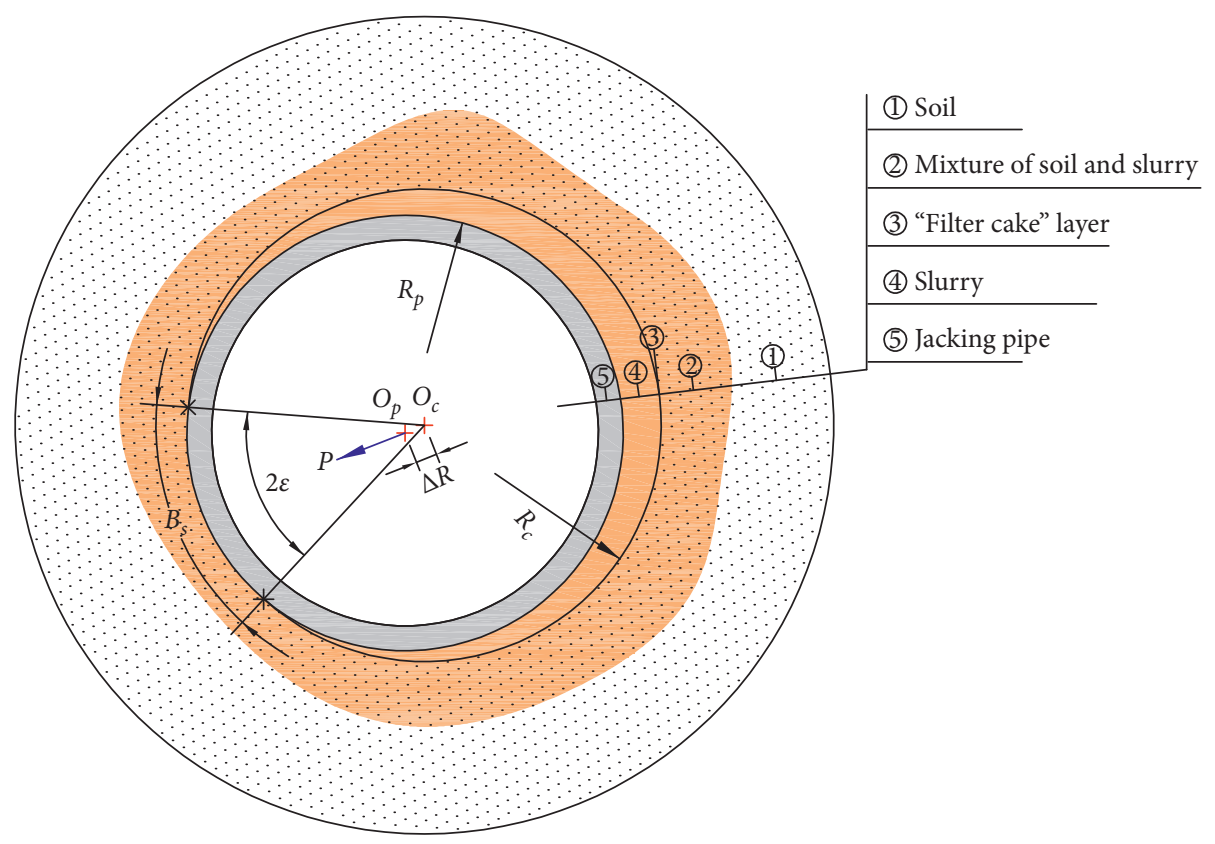

FIgURE 4: Contact model and symbols used.

By substituting (9) and (10) into (8), after some algebra, (12) can be further rewritten as

$$
\mu=\mu_{s} \frac{\varepsilon}{\pi}+\mu_{m}\left(1-\frac{\varepsilon}{\pi(1+e)}\right)
$$

From (15), the key to calculate the effective friction coefficient $\mu$ is to calculate the width (or angle) of contact. It has to be noted that at the present time by far the most used model is the Hertz contact model $[7,8]$. The contact width is given by

$$
\begin{aligned}
B_{s} & =1.6\left(P k_{d} C_{e}\right)^{1 / 2}, \\
k_{d} & =\frac{D_{c} D_{p}}{D_{c}-D_{p}}, \\
C_{e} & =\frac{1-v_{p}^{2}}{E_{p}}+\frac{1-v_{s}^{2}}{E_{s}},
\end{aligned}
$$

where $D_{c}$ and $D_{p}$ are the internal diameter of cavity and external diameter of pipe, respectively, $v_{p}$ and $v_{s}$ are Poisson's ratio of the pipe and soil material, respectively, $E_{p}$ and $E_{s}$ are the elastic modulus for pipe and soil material, respectively, and $P$ is the external force acting on the center of the pipe. If the excavation cavity is stable, the pipe is in contact with the cavity at the bottom due to its own weight, and $P$ is equal to the weight of pipe per unit length $[5,7]$. For slurry pipe jacking, according to hypothesis (ii), $P$ is approximately equal to the total Earth pressure at contact area; it then gives

$$
P=\frac{\varepsilon}{\pi} N
$$

Hertzian model provides a simple way for the calculation of the width of contact; however, the Hertzian contact problem is approached only when the applied force is small or the large radial clearance is large, and the limited angle of contact is smaller than about $30^{\circ}$ [12]. Due to the technical limitations, most of the pipe jacking projects encounter clay or sandy soils with small radial clearance; it is therefore that the applicability of Hertz contact model is extremely limited here. Actually, the Hertz contact model is just a special case of the Persson contact model with a small contact width (or angle) [12]. If a large possible contact angle (larger than $30^{\circ}$ ) happens, the more general contact model proposed by Persson should be taken as the first consideration. For simple, the approximate form for the contact angle relation put forward by Michele and Paolo [17], from Persson model, is used in this paper. The expression is given by

$$
\frac{\pi(\alpha+1) E_{p} \Delta R}{\left(1-v_{p}^{2}\right) P}=\frac{(\alpha-1)\left[\ln \left(\xi^{2}+1\right)+2 \xi^{4}\right]+2}{\left(\xi^{2}+1\right) \xi^{2}}-4 \beta
$$

$$
\begin{aligned}
\Delta R & =\frac{D_{c}-D_{p}}{2}, \\
\xi & =\tan \left(\frac{\varepsilon}{2}\right), \\
\eta & =\frac{E_{p}}{E_{s}} \frac{1-v_{s}^{2}}{1-v_{p}^{2}} \\
\lambda & =\frac{1-2 v_{p}}{1-v_{p}}-\eta \frac{1-2 v_{s}}{1-v_{s}}, \\
\alpha & =\frac{1-\eta}{1+\eta} \\
\beta & =\frac{\lambda}{2(1+\eta)} .
\end{aligned}
$$

As comparison with (16), (19) is a more complex nonlinear equation. It can be further simplified with respect to the actual situation that the elastic modulus of soil $E_{s}$ is much smaller than that of pipe $E_{p}$ (the difference between the two 
is usually three orders of magnitude). Thus, from (20), the value of auxiliary variable $\eta$ should be very large, and the approximate relations can be obtained as

$$
\begin{aligned}
\frac{\pi(\alpha+1) E_{p}}{\left(1-v_{p}^{2}\right)} & \approx \frac{2 \pi E_{s}}{\left(1-v_{s}^{2}\right)}, \\
\alpha & \approx-1, \\
\beta & \approx \frac{1-2 v_{s}}{2\left(1-v_{s}\right)} .
\end{aligned}
$$

Using (21), (19) is simplified as

$$
\frac{\pi E_{s} \Delta R}{\left(1-v_{s}^{2}\right) P}+\frac{1-2 v_{s}}{1-v_{s}}=\frac{1-\left[\ln \left(\xi^{2}+1\right)+2 \xi^{4}\right]}{\left(\xi^{2}+1\right) \xi^{2}} \text {. }
$$

Together with (5), (15), (18), and (22), the contact angle $2 \varepsilon$, the effective friction coefficient $\mu$, and the friction force $F_{f}$ now can be uniquely identified. Apparently, the effective friction coefficient here is not just related to the interfriction angle of soil $\varphi$ but the other soil parameters $\left(E_{s}, v_{s}\right.$, and $e$ ) and design parameters $\left(h, D_{p}\right.$, and $\left.\Delta R\right)$. That is to say, for a specific pipe jacking project, the effective friction coefficient is probably not a constant for the complex geological conditions.

\section{Comparison between the Predicted Friction and the Measured Friction}

Ten slurry pipe jacking projects with 12 measured data were collected from literature $[5-7,18,19]$, to check with the predicted result of the model. These projects encountered some representative soils such as sandy clay, silt, sand and gravels. Also, they have different overburden depth of 5-12 m, radial clearance of $0-30 \mathrm{~mm}$, and pipe diameters of 0.5-4.14 m (see in Table 3). In particular, Cases 11-12 were in the condition of water rich for passing through a river. These characteristics of the projects provide good foundation for evaluating the capability of the model.

Some parameters that needed to calculate the predicted equations were not given in the literature. So the values of geological parameters involved in the new model (taken from the Geological Engineering Handbook [20]) are summarized in Table 4 . In principle, during the calculation, the parameters given in the in situ case should be used, and the missing parameters can be selected from Table 4 . Therefore, the parameters in each case were finally determined and summarized in Table 3.

Frequently, some parameter given is a value range, rather than a specific number. Thereby, it faces a problem of parameter combination to calculate the maximum and minimum friction force. Accordingly, the relationships between various parameters and the calculated friction force were studied first by single-factor analysis; the results have been shown in Table 5.

In Table 5, the symbol "+" indicates that the relationship between the two is positively correlated and the symbol "-" indicates that they are negatively correlated. When the maximum friction force is to be calculated, the quantities of negative correlation should be the minimums, while the quantities of positive correlation should be the maximums. And for the calculation of minimum friction force, the opposite is true.

In Table 1, for each of the drives, measured frictional force values are presented and compared to values calculated by the three approaches of Terzaghi's initial model and the two modified models. One can see that most of the in situ results are included in the predicted range of values calculated by PJA (UK) and AVTA (Germany) model, respectively, suggesting that both of them are capable of accurately calculating the friction resistance of slurry pipe jacking. And the frictions calculated by AVTA (Germany) model are slightly larger than that calculated by PJA (UK) model, which is explained by the different parameters $b, K$, and $\delta$ used (see in Table 2).

Despite overall poor performance (much smaller predictions) for Terzaghi's initial model, it makes even better predictions in Cases 11-12 (especially in Case 11) which drive under a river. It may indicate that, in the condition of water rich, the boundary planes of wedge failures (a bigger $b$ ) assumed by Terzaghi are more consistent with the actual situation.

The calculation results of the contact angle and the corresponding effective friction coefficient in each case are also given in Table 1. According to the calculation results, the friction coefficient of slurry pipe jacking may be $0.01-0.16$, which is almost the same as the result $0.03-0.13$ acquired by backcalculation with Terzaghi initial silo model [5]. Special Case 5 is with radial clearance $\Delta R=0$, which makes the calculated contact angle as high as $130^{\circ}$, indirectly leading to a large friction coefficient of 0.16 . Apart from this case, most of effective friction coefficients vary between 0.02 and 0.1 .

It is noted that Case 4 and Case 5 have almost the same geological conditions and design parameters except for the radial clearance (Case 4 is $20 \mathrm{~mm}$ and Case 5 is $0 \mathrm{~mm}$ ). And the calculated friction in Case 5 is consistent with the measured value, while that in Case 4 is much smaller (see in Table 1). However, if we reset $\Delta R$ in Case 4 as 0 , using ATVA model, the recalculated friction is $7.48-10.48 \mathrm{kN} /$ $\mathrm{m}$, which is then consistent with the measured value $(9.55 \mathrm{kN} / \mathrm{m})$. One explanation is that the amount of grouting in Case 4 may be insufficient, causing the soil relaxation, and fill the whole annular space. Another more plausible explanation here is that, in sand and gravels with large voids, the injected slurry soon penetrates into the soil accompany with pressure dissipation, and the soil then comes into full contact with the pipe. In addition, the calculation of Case 10 with similar strata (drive in sand and gravels under a river) does not encounter the same problem as that in Case 4. It suggests that, under the condition of water rich, volts in soil are completely filled with water, so the interpenetration between the injected slurry and soil does not occur notably; thereby, the pressure of the injected slurry is sufficient to keep the annular space open and stable. 


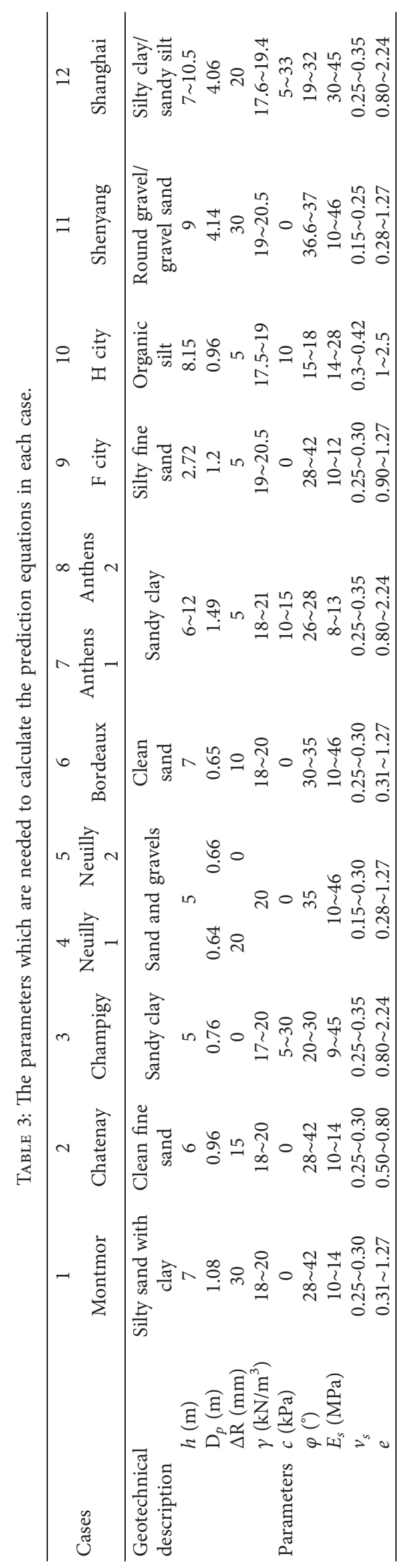


TABLE 4: The general geotechnical parameters.

\begin{tabular}{lcccccc}
\hline $\begin{array}{l}\text { Soil } \\
\text { group }\end{array}$ & $\begin{array}{c}\gamma \\
\left(\mathrm{kN} / \mathrm{m}^{3}\right)\end{array}$ & $\varphi\left(^{\circ}\right)$ & $\begin{array}{c}c \\
(\mathrm{kPa})\end{array}$ & $\begin{array}{c}E_{s} \\
(\mathrm{MPa})\end{array}$ & $v_{s}$ & $e$ \\
\hline $\begin{array}{l}\text { Gravel } \\
18.7 \sim 22.8\end{array}$ & $33 \sim 45$ & 0 & $14 \sim 42$ & $0.15 \sim 0.25$ & $0.28 \sim 0.62$ \\
$\begin{array}{l}\text { Sand } \\
\text { Clayey }\end{array}$ & $19 \sim 20.5$ & $28 \sim 42$ & 0 & $10 \sim 46$ & $0.25 \sim 0.35$ & $0.31 \sim 1.27$ \\
$\begin{array}{l}\text { sand } \\
\text { Sandy }\end{array}$ & $19.5 \sim 21$ & $13 \sim 30$ & $2 \sim 7$ & $11 \sim 23$ & $0.30 \sim 0.40$ & $0.41 \sim 1.96$ \\
$\begin{array}{l}\text { clay } \\
\text { Clay }\end{array}$ & $18 \sim 21$ & $17 \sim 24$ & $5 \sim 40$ & $9 \sim 45$ & $0.25 \sim 0.35$ & $0.80 \sim 2.24$ \\
\hline
\end{tabular}

TABLE 5: The relationship between the parameters and the calculated friction.

\begin{tabular}{cccccccccc}
\hline & Models & $\gamma$ & $\varphi$ & $c$ & $E_{s}$ & $v_{s}$ & $e$ & $D_{e}$ & $h$ \\
\hline \multirow{2}{*}{$F_{f}$} & Terzaghi & + & - & - & - & + & + & + & + \\
& PJA and ATVA & + & + & - & - & + & + & + & + \\
\hline
\end{tabular}

\section{Conclusions}

This paper presents a new method for the calculation of friction resistance for slurry pipe jacking. Good predictions were made in 12 in situ cases with various soils and design parameters. The following conclusions in the calculation can be drawn:

(1) In most cases, assuming a big influencing width of soil above the pipe of Terzaghi initial silo model leads to an underestimation of the soil pressure, and the modified models proposed by PJA (UK) and ATVA (Germany) seem more realistic. And under the condition of water rich, Terzaghi initial silo model performs even better, which may need more cases to check out.

(2) The effective friction coefficient for slurry pipe jacking, taking the approach of ATVA model for example, is mostly ranging from 0.02 to 0.1 , depending on the contact state of pipe soil slurry, which is not only affected by geological parameters, but also closely related to design parameters, such as buried depth, pipe diameter, and radial clearance (overcut).

(3) The overcut of design determines the volume of injected lubricant slurry; its value has a significant influence on the effective friction coefficient and therefore the friction resistance. In some strata such as sand and gravels, the injected slurry may not create a screen to keep the excavated bore stable. In this case, the effect of overcut can be underestimated or even ignored to get a better prediction result.

\section{Abbreviations}

$F_{f}$ : Friction force per meter length drive

$\mu$ : $\quad$ Effective friction coefficient for slurry pipe jacking

$\mu_{s}: \quad$ Soil-pipe friction coefficient $\mu_{m}:$ Slurry-pipe friction coefficient

$N$ : Normal force due to ground pressure acting on pipe

$\sigma_{n}$ : Normal soil stress acting on any point of pipes

$\sigma_{v}:$ Vertical soil stress

$\sigma_{h}:$ Horizontal soil stress

$D_{c}$ : Internal diameter of cavity

$D_{p}$ : External diameter of pipe

$b$ : Influencing width of soil above the pipe ideal silo width

c: Soil cohesion

$\varphi: \quad$ Inner friction angle of soil

$\delta$ : $\quad$ Soil-pipe friction angle

$\gamma$ : Unit weight of soil

$e: \quad$ Void ratio of soil

$K$ : Coefficient of soil pressure above the pipe

$k$ : Terzaghi coefficient which represents the "vault" effect of the ground

$h$ : Height of cover at pipe crown

$\varepsilon$ : Semiangle of contact area

$B_{s}$ : Width of contact area between the pipe and soil

$R_{c}$ : Internal radius of cavity

$R_{p}$ : External radius of pipe

$\Delta R$ : Radial clearance (or overcut)

$E_{p}$ : Elasticity modulus of pipe

$E_{s}$ : Elasticity modulus of soil

$v_{p}$ : Poisson's ratio of pipe

$v_{s}$ : Poisson's ratio of soil

$P$ : External load applied at the center of the pipes.

\section{Data Availability}

All the measured data and calculation results data used to support the findings of this study are available and included within the article.

\section{Conflicts of Interest}

The authors declare no conflicts of interest.

\section{Acknowledgments}

The authors acknowledge the financial support of the National Natural Science Foundation of China (no. 51878670).

\section{References}

[1] D.-J. Ren, Y.-S. Xu, J. Shen, A. Zhou, and A. Arulrajah, "Prediction of ground deformation during pipe-jacking considering multiple factors," Applied Sciences, vol. 8, no. 7, p. 1051, 2018

[2] Y. Zhang, Z. G. Yan, and H. H. Zhu, "A Full-Scale Experimental study on the performance of jacking prestressed concrete cylinder pipe with misalignment angle," Proceedings of GeoShanghai 2018 International Conference: Multi-physics Processes in Soil Mechanics and Advances in Geotechnical Testing, Springer, Singapore, pp. 345-354, 2018.

[3] S. Khazaei, H. Shimada, T. Kawai, J. Yotsumoto, and K. Matsui, "Monitoring of over cutting area and lubrication distribution in a large slurry pipe jacking operation," Geotechnical and Geological Engineering, vol. 24, no. 3, pp. 735755, 2006. 
[4] X. Yang, Y. Liu, and C. Yang, "Research on the slurry for longdistance large-diameter pipe jacking in expansive soil," $A d$ vances in Civil Engineering, vol. 2018, Article ID 9040471, 9 pages, 2018.

[5] A.-L. Pellet-Beaucour and R. Kastner, "Experimental and analytical study of friction forces during microtunneling operations," Tunnelling and Underground Space Technology, vol. 17, no. 1, pp. 83-97, 2002.

[6] S. Hideki, K. Saeid, and M. Kikuo, "Small diameter tunnel excavation method using slurry pipe-jacking," Geotechnical and Geological Engineering, vol. 22, no. 2, pp. 161-186, 2004.

[7] A. I. Sofianos, P. Loukas, and C. Chantzakos, "Pipe jacking a sewer under Athens," Tunnelling and Underground Space Technology, vol. 19, no. 2, pp. 193-203, 2004.

[8] G. W. E. Milligan and P. Norris, "Site-based research in pipe jacking-objectives, procedures and a case history," Tunnelling and Underground Space Technology, vol. 11, pp. 3-24, 1996.

[9] C. Michele and D. Paolo, "The state of stress induced by the plane frictionless cylindrical contact. I. The case of elastic similarity," International Journal of Solids and Structures, vol. 38, no. 26-27, pp. 4507-4523, 2001.

[10] D. Stein, K. Mollers, and R. Bielecki, Microtunnelling, p. 352, W. Ernst und Sohn, Berlin, Germany, 1989.

[11] K. J. Shou and M. J. Jiang, "A study of jacking force for a curved pipejacking," Journal of Rock Mechanics and Geotechnical Engineering, vol. 2, no. 4, pp. 298-304, 2010.

[12] M. Barla, M. Camusso, and S. Aiassa, "Analysis of jacking forces during microtunnelling in limestone," Tunnelling and Underground Space Technology, vol. 21, no. 6, pp. 668-683, 2006.

[13] D. N. Chapman and Y. Ichioka, "Prediction of jacking forces for microtunnelling operations," Tunnelling and Underground Space Technology, vol. 14, no. 1, pp. 31-41, 1999.

[14] K. Shou, J. Yen, and M. Liu, "On the frictional property of lubricants and its impact on jacking force and soil-pipe interaction of pipe-jacking," Tunnelling and Underground Space Technology, vol. 25, no. 4, pp. 469-477, 2010.

[15] G. W. E. Milligan and P. Norris, "Pipe-soil interaction during pipe jacking," Proceedings of the Institution of Civil Engineers-Geotechnical Engineering, vol. 137, no. 1, pp. 27-44, 1999.

[16] W. Guo, H. Xie, R. Wu, and B. Zhou, "Experimental study on bentonite lubrication during pipe jacking construction," Journal of Henan Science and Technology, vol. 555, no. 1, pp. 115-118, 2015, in Chinese.

[17] C. Michele and D. Paolo, "The state of stress induced by the plane frictionless cylindrical contact. II. The general case (elastic dissimilarity)," International Journal of Solids and Structures, vol. 38, no. 26-27, pp. 4523-4533, 2001.

[18] J. Wang, K. Wang, T. Zhang, and S. Wang, "Key aspects of a DN4000 steel pipe jacking project in China: a case study of a water pipeline in the Shanghai Huangpu river," Tunnelling and Underground Space Technology, vol. 72, pp. 323-332, 2018.

[19] X.-B. Ji, W. Zhao, P. Jia et al., "Pipe jacking in sandy soil under a river in Shenyang, China," Indian Geotechnical Journal, vol. 47, no. 3, pp. 246-260, 2017.

[20] Z. H. Shi, M. Wang, D. Q. Xian, and Y. Yang, Geological Engineering Handbook, pp. 174-179, China Building Industry Press, Beijing, China, 2018. 